\title{
SPRACH-FLUSS: Theaterworkshops mit Jugendlichen aus 16 afrikanischen Ländern - Theaterpädagogik zwischen kultureller Bildung und Fremdsprachendidaktik
}

\author{
Edda Holl
}

\begin{abstract}
Zusammenfassung
Im Rahmen der Initiative der Bundesrepublik Deutschland 'Schulen: Partner der Zukunft' (PASCH) initiierte, konzipierte und leitete die Autorin im Auftrag des Goethe-Instituts SPRACH-FLUSS, das umfangreichste PASCH-Projekt in Subsahara-Afrika. In Teams zu viert leiteten Theaterpädagoginnen und -pädagogen der Universität Hildesheim insgesamt acht Theaterworkshops, bei denen jeweils eine vielsprachige Präsentation erarbeitet wurde. Parallel zu den Theaterwerkstätten wurden mit ebenfalls daran teilnehmenden Deutschlehrkräften aus Afrika und Deutschland einzelne theaterpädagogische Übungen und Verfahren ausgewählt und für den Deutschunterricht abgewandelt. In diesem Artikel wird das Projekt SPRACH-FLUSS vorgestellt, vor allem wie die Theaterpädagogen die Jugendlichen, die ganz verschiedenen sprachlichen und kulturellen Hintergründen entstammten, zu einem Ensemble zusammenführten. Weiterhin wird gezeigt, wie Ausrichtung und Zweck einzelner theaterpädagogischer Übungen sich verändern, wenn diese im gesteuerten Fremdsprachenunterricht eingesetzt werden.
\end{abstract}

\section{SPRACH-FLUSS}

SPRACH-FLUSS ist der Name des größten PASCH ${ }^{1}$-Projektes der Bundesrepublik Deutschland in Subsahara-Afrika. Von September 2008 bis September 2009 reisten insgesamt 13 Theaterpädagogen/innen zu Standorten des GoetheInstituts in Namibia, Togo, Ghana, Uganda, Kenia und Südafrika, um dort in Teams zu viert mehrsprachige Theaterworkshops zu leiten. Bei jedem Workshop begegneten sich 20 Schülerinnen und Schüler im Alter zwischen 14 und 18 Jahren und jeweils vier Deutschlehrkräfte. Die Jugendlichen kamen von 34 PASCH-Schulen aus 16 frankophonen und anglophonen Ländern 
Afrikas, ${ }^{2}$ und da die meisten von ihnen bereits drei bis fünf Sprachen gelernt hatten, gehörte Mehrsprachigkeit zur ihrer kulturellen Identität. Die Relaissprache bei der theaterpädagogischen Arbeit war Deutsch, wobei die Deutschkenntnisse der Jugendlichen sehr variierten: einige hatten bereits vier Jahre Deutsch als Fremdsprache gelernt, andere lediglich sechs Monate. Bei den fünf einführenden Theaterwerkstätten wurden den Jugendlichen kommunikative und theatrale Mittel an die Hand gegeben, mit denen sie selbst Szenen entwickelten. So entstanden szenische Präsentationen, die Erfahrungen, Hoffnungen, Enttäuschungen und Visionen der Teenager ausdrückten und über die sie sich miteinander austauschten. Eine Auswahl dieser Jugendlichen nahm im Mai an einem panafrikanischen Workshop in Südafrika teil, der sie auf einen Workshopaufenthalt in Deutschland vorbereitete. Die Sprachund Kommunikationswerkzeuge, die sie dabei kennengelernt und erprobt hatten, konnten die afrikanischen Jugendlichen in dem abschließenden Begegnungsworkshop mit deutschen Schülerinnen und Schülern erfolgreich einsetzen. Dieser Brandenburger Workshop fand seinen Höhepunkt am 18. September 2009 in einer mehrsprachigen Bühnenpräsentation in der Akademie der Künste, Berlin im Rahmen der Veranstaltung, „Sprachen ohne Grenzen“.

\section{Der Auftrag des Goethe-Instituts}

Das Goethe-Institut verfolgte mit diesem PASCH- Projekt verschiedene Ziele: Die Schülerinnen und Schüler der Partnerschulen aus der gesamten Region Subsahara- Afrika sollten sich kennenlernen, miteinander vernetzt werden und dabei die deutsche Sprache als internationales Kommunikationsmittel erleben. Die Überzeugung vieler Jugendlicher, Deutschen sei eine kaum zu erlernende Sprache, galt es durch positive, motivierende Erfahrungen während der Workshops zu entkräften. Denn obwohl die afrikanischen Jugendlichen bereits etliche der lokalen Kommunikationssprachen erfolgreich erlernt haben, fühlen sich viele von ihnen im Deutschunterricht überfordert. Die Ursache dafür liegt vermutlich nicht darin, dass Deutsch eine besonders komplexe Sprache ist, sondern eher am abrupten Wechsel vom selbst gesteuerten, beiläufigen Spracherwerb in der regionalen Immersion zum bewussten Sprachlernen im Klassenraum, das die Schüler im Deutschunterricht zum ersten Mal erleben (cf. Sarter 1997: 26ff). Ausgehend von Erkenntnissen der Mehrsprachigkeitsforschung, nach denen jeder neue Sprachlernprozess sich auf bereits erlernte Sprachen stützt (cf. Hufeisen 2008), interessieren sich die

\footnotetext{
${ }^{2}$ Auf dem afrikanischen Kontinent werden ca. 1500 untereinander nicht verständliche Sprachen gesprochen. Je nach der kolonialen Sprache, die zur offiziellen Landessprache erhoben wurde, spricht man von frankophonen, anglophonen bzw. lusophonen Ländern. Dass im Curriculum der frankophonen Länder lange Zeit Deutsch vor Englisch als Fremdsprache eingeführt wurde, ist noch heute spürbar: Fast zwei Drittel der an SPRACH-FLUSS beteiligten PASCHSchulen befinden sich in der Frankophonie. In lusophonen Ländern wie Angola oder Mosambik existierten 2009 noch keine PASCH-Schulen, weshalb keine portugiesischsprachigen Jugendlichen am Projekt teilnahmen.
} 
Fremdsprachendidaktiker des Goethe-Instituts besonders für die Frage, wie sich die Mehrsprachigkeit der afrikanischen Schüler für den Deutschunterricht nutzen lässt. Aus diesem Anliegen erklärt sich die Empfehlung des GoetheInstituts an die Theaterpädagogen des PASCH-Projekts, sie sollten bei den Workshops der Mehrsprachigkeit der Jugendlichen 'viel Raum' geben.

Vom PASCH-Projekt profitieren sollten auch die Deutschlehrkräfte der Region. Aufgrund hoher Klassenstärken - in Westafrika sind es bis zu 120 Lernende pro Klasse - überwiegt ein auf grammatischen Regeln und Vokabellernen basierender Frontalunterricht. Deshalb wurde seitens des Goethe-Instituts angeregt, 20 Lehrkräfte als Teilnehmende, Beobachtende und Beratende in das Projekt einzubeziehen. Sie sollten den theaterpädagogischen Prozess erleben und gemeinsam mit den Theaterpädagogen Verfahren und Mittel erproben, durch die sich Deutschunterricht lebendiger, lustvoller und effektiver gestalten lässt.

Schließlich wollten die Zuständigen des Goethe-Instituts die Nachhaltigkeit des Projekts sichern: Um auch denjenigen PASCH-Schülern und Lehrern, die nicht an einem Workshop teilnehmen würden, die theaterpädagogische Arbeit vorzustellen, beauftragte man ein Filmteam damit, alle acht Workshops zu dokumentieren und einen SPRACH-FLUSS-Film herzustellen. Die Filmemacherin hatte darüber hinaus den Auftrag, diejenigen theaterpädagogischen Übungen und Verfahren aufzuzeichnen, die nach Auffassung der Lehrkräfte im Unterricht einsetzbar wären. Diese Filmaufnahmen illustrieren das 2011 erschienene SPRACH-FLUSS Handbuch, in dem die wichtigsten, für den (Sprach-) Unterricht relevanten theaterpädagogischen Mittel aus dem Projekt beschrieben und erläutert sind (cf. Holl 2011).

\section{Vorbereitungen des theaterpädagogischen Teams}

Im Frühjahr 2008 konzipierte die Projektleiterin das acht Workshops umfassende Projekt SPRACH-FLUSS, und stellte es den Experten für Unterricht vor, die an den Goethe-Instituten der Region für die Betreuung der PASCH-Schulen zuständig sind.

Um die Workshops gemeinsam zu planen, traf sich das theaterpädagogische Team im Juni 2008 - also drei Monate vor Beginn der ersten Werkstatt - zu einem fünftägigen Vorbereitungsseminar in Hildesheim. Die Lehrenden, Absolventen und Studierenden der Universität Hildesheim verfügten über vielfältige theaterpädagogische Erfahrungen: Viele hatten Inszenierungen im Bereich des Kinder- und Jugendtheaters entwickelt, und alle hatten theaterpädagogische Prozesse geleitet, sei es an Schulen, in Stadttheatern, in Stadteilen mit sozialen Brennpunkten, in Jugendeinrichtungen oder kulturellen Bildungsstätten. In einem afrikanischen Land hatte jedoch noch keiner von ihnen gearbeitet.

Nach der Klärung von organisatorischen Fragen wie Reiserouten, Visumsbeschaffung, Impfungen, Malariaprophylaxe und Workshopleitung konnte die inhaltliche Planung beginnen: Es wurde dabei zunächst diskutiert, wie die afrikanischen Jugendlichen einander kennenlernen, miteinander vertraut gemacht 
und mit ihren verschiedenen Sprachen gemeinsam ins Spiel gebracht werden sollten. Darüber hinaus hatten alle Theaterpädagogen Übungen ausgewählt und sich szenische Spielaufgaben ausgedacht, die sie für die afrikanischen Workshops für geeignet hielten.

Abwechselnd leiteten die Theaterpädagogen ihre Übungen an, wobei die Gruppenmitglieder versuchten, die Workshopsituation mit mehrsprachigen Jugendlichen in Afrika zu antizipieren. So wurde z.B. streng darauf geachtet, deutlich und langsam in Übungen einzuweisen und eindeutige Gesten einzusetzen. Dabei wurde auch erkannt, wie wichtig es ist, Übungen gleich in mehrfachen Haltungen vorzumachen, da die Lernenden sonst davon ausgehen könnten, dass es lediglich eine richtige Art des Agierens gäbe. Beim anschließenden Auswerten der praktischen Vorschläge bemerkten etliche Theaterpädagogen, dass es zur angemessenen Vorbereitung näherer Informationen über die Jugendlichen, insbesondere bezüglich ihrer Deutschkenntnisse, ihrer Schicht- und Religionszugehörigkeit sowie ihrer Lebensbedingungen bedürfe. Derlei Auskünfte waren leider nicht zu ermitteln, schließlich handelte es sich bei den PASCH-Schülern um Jugendliche aus 34 verschiedenen Schulen in 16 afrikanischen Ländern.

\section{Theaterpädagogik und selbstgesteuerte Sprachlernprozesse}

Da keiner der Theaterpädagogen über eine DaF- oder DaZ-Ausbildung verfügte und nur wenige bereits mit nicht-deutsch sprechenden Jugendlichen gearbeitet hatten, kamen in dieser Phase der Workshopvorbereitungen etliche Bedenken auf: „Werden wir uns mit den afrikanischen Jugendlichen hinreichend verständigen können, um ein Vertrauensverhältnis zu ihnen aufbauen zu können? Lässt sich eine vielsprachige Gruppe in so kurzer Zeit über die szenische Arbeit zu einem Ensemble zusammenführen? Müssen wir den Teenagern Deutsch beibringen?" und „Wie können wir die Mehrsprachigkeit der Jugendlichen anerkennen und würdigen, wenn wir deren Sprachen selbst nicht sprechen?" waren Fragen aus dem Team, die grundsätzlicher Klärung bedurften, damit überhaupt eingeschätzt werden konnte, welche der vorgeschlagenen Übungen und Verfahren im Kontext von SPRACH-FLUSS sinnvoll und dienlich sein würden.

Eine Antwort auf die Frage, in welcher Weise Theaterpädagogen ohne fremdsprachendidaktische Vorkenntnisse dennoch Sprachlernen fördern können, fand sich bei der US-amerikanischen Forscherin Joan Rubin, die sich bereits in den 70er Jahren des letzten Jahrhunderts entschieden dafür eingesetzt hatte, dass Sprachlernprozesse vor allem auch aus der Perspektive der Lernenden untersucht werden müssten. Auf Hawaii beobachtete sie vor 37 Jahren unter der Fragestellung: „What the 'Good Language Learner' Can Teach Us?" (Rubin 1975) den selbst gesteuerten Sprachlernprozess von Einwandererkindern und stellte heraus, welche sozialen Fähigkeiten und Strategien diejenigen Schüler 
und Studenten anwendeten, die am besten und schnellsten Englisch als Zweitbzw. Drittsprache lernten. Für Rubin gehört dazu,

- sich einer Gruppe anzuschließen und so zu tun, als ob man wüsste, worüber gesprochen wird, auch wenn man es nicht weiß;

- sich mit wenigen Floskeln den Anschein zu geben, als ob man die Sprache beherrschte;

- mit der Hilfe seiner Freunde zu rechnen;

- dieBereitschaft, gutund präzise zu raten und eigenes Wissen einzubringen, um die Bedeutung von Wörtern oder Satzstrukturen zu entschlüsseln;

- zeitweise mit dem Gefühl der Ungewissheit leben zu können, dabei jedoch ständig bemüht zu sein, im weiteren Verlauf der Kommunikation die eigenen Annahmen zu überprüfen und zu korrigieren;

- ein großes Kommunikations- und Lernbedürfnis zu haben, um die Erfolgschancen der Kommunikation zu erhöhen, kreativ mit geringen Redemitteln umzugehen, unbekannte Vokabeln zu umschreiben;

- Fantasie zu entwickeln und auf persönliche Eitelkeit zu verzichten, also keine Angst zu haben, sich durch falsche Aussprache, Fehler oder durch das Anwenden von Körpersprache zu blamieren;

- die Kommunikation mit Muttersprachlern zu suchen und jede Gelegenheit zu nutzen, um Sätze und Redewendungen zu üben, die man sich zurechtgelegt hat;

- Sprachbewusstheit zu entwickeln d. h. jeweils genau zu wissen, wann man mit wem welche Sprache in welchem Duktus spricht. Dazu gehört auch das Sprachverhalten von Gesprächspartnern zu erfassen und auf neue Redewendungen hin abzuklopfen;

- sich durch eigene sprachliche Unzulänglichkeiten nicht entmutigen zu lassen, sondern aus missglückten Kommunikationshandlungen zu lernen;

- über grammatische Aufmerksamkeit für Formen und Strukturen der fremden Sprache zu verfügen; beständig zu versuchen, Sprachmuster zu erkennen, sie zu kategorisieren und anzuwenden.

,Gute Sprachlernende' nehmen also ihren Lernprozess selbst in die Hand und bringen dabei ihr Wissen und ihre Persönlichkeit umfassend ein. Sie verbinden Elemente des bewussten Sprachlernens, wie z.B. das Erfassen von grammatischen Regeln mit Elementen des unbewussten, beiläufigen Spracherwerbs, etwa dem intuitiven, präzisen Raten. Die Workshopleiter erkannten, dass diejenigen sozialen und kommunikativen Kompetenzen, die gemäß Rubin das selbständige Sprachlernen fördern, weitgehend identisch sind mit den Fähigkeiten und Fertigkeiten, die in der theaterpädagogischen Arbeit und im Darstellenden Spiel ausgebildet und kultiviert werden, nämlich: 
- Gesprächsbeziehungen zu initiieren und aufrechtzuerhalten;

- sich auf Kommunikationspartner zu verlassen;

- so zu tun, als ob man eine Sprache spricht, indem man kreativ mit begrenzten Redemitteln umgeht;

- keine Scheu zu haben, Körpersprache einzusetzen, um sich verständlich zu machen;

- die soziale Beziehung der jeweiligen Sprecher mitzudenken und auch Subtext, also das, was unter dem verbal Geäußerten liegt, gut lesen zu können.

Ausgehend von der These, dass jede gute theaterpädagogische Übung auch eine gute Kommunikationsübung sei und ihre Anwendung insofern Sprachlernkompetenzen ausbilden würde, machten sich die Theaterpädagogen nun daran, die aus ihrem Kreis vorgeschlagenen Übungen hinsichtlich ihrer Relevanz für das Sprachenlernen zu untersuchen. Einzelne Techniken und Herangehensweisen wurden dabei ausprobiert, evaluiert und folgender Typologie zugeordnet:

- Rhythmus und Mnemotechniken, um intuitiv in den SPRACH-FLUSS zu kommen, aber auch um lustvoll auswendig zu lernen, Sprachgefühl zu entwickeln und sich dabei das Gedächtnis des Körpers zunutze zu machen.

- Assoziationsübungen, um ein intuitives Sprachgefühl jenseits von ,richtig und falsch`zu entwickeln und auch die eigenen Gedächtnisspeicher zu entdecken und im Lernprozess nutzen zu können. Ferner lernt man die eigenen Ideen, Gedanken und Vorstellungen ernst $\mathrm{zu}$ nehmen. $\mathrm{Zu}$ bestimmten Kontexten gemeinsam $\mathrm{zu}$ assoziieren und Wortfelder aufzurufen, schult das Verknüpfen von Wissen aus verschiedensten Disziplinen und Lebensbereichen und fördert damit das präzise Raten.

- Impulsübungen, um in Gesprächssituationen adäquat reagieren zu können: sich zu trauen, beherzt zu agieren, auf Gesprächspartner fokussiert zu sein, klar zu adressieren und Kommunikationsangebote zu erkennen und zu nutzen.

- Körperübungen und Massagen, um Körperbewusstheit zu erlangen und nonverbale Kommunikation einzusetzen, Blockaden zu lösen und mit allen Sinneskanälen wahrzunehmen, Körper und Geist sozusagen auf ,Empfangsmodus‘ für Neues einzustellen.

- Partnerübungen/ sich visuell und akustisch Einfühlen, umsehr unterschiedliche Kommunikationspartner ,lesen' zu können und jeweils individuelle Beziehungen zu ihnen herstellen zu können. Auch um Gesprächskontakte zu initiieren und aufrechtzuerhalten. 
- Improvisationen/Szenen erarbeiten, um zu üben, vor der Gruppe eigene Ideen und Vorschläge zu verbalisieren, gemeinsame Aufgaben und Problemstellungen $\mathrm{zu}$ formulieren und das Arbeitsprinzip des gemeinsamen Ausprobierens, Besprechens und Weiterentwickelns an einem sinnlich Wahrnehmbaren zu üben.

- Präzises Beobachten/Zuschaukunst um anschauliches Beschreiben (möglichst auf Deutsch) zu schulen und zu kultivieren; und einen analytischen Blick auf Situationen, Interaktionen und Beziehungen zwischen Figuren - bzw. Menschen im Alltag - zu entwickeln. ${ }^{3}$

Die Theaterpädagogen hatten nun eine viel klarere Vorstellung davon, wie sie bei den Workshops durch ihre theaterpädagogischen Mittel das selbstgesteuerte Sprachlernen der PASCH-Schüler fördern könnten. Aber damit war nur ein Teil der Bedenken in der Teamrunde ausgeräumt. Auch wenn es die Sprachlernprozesse der einzelnen Schüler unterstützte, entsprach es nicht dem theaterpädagogischen Selbstverständnis der meisten Anwesenden „mit dem theaterpädagogischen Hebammenkoffer nach Afrika zu gehen und den Jugendlichen bloß Übungen beizubringen und sie mit szenischen Verfahren bekannt zu machen", wie ein Theaterpädagogin es formulierte. Die Vorstellung, während der Workshops lediglich verschiedene Übungstypen anzuleiten und aus den Ergebnissen eine Szenenfolge zu generieren, ohne dass die Jugendlichen sich angemessen einbringen könnten, erachteten einige Teammitglieder als eine Form von Kulturkolonialismus. Auch wenn das SPRACH-FLUSS Projekt dazu animiert habe, sich über verschiedene Länder Afrikas zu informieren, hatten die Meldungen zu Themen wie Bildungssysteme, die Ausbreitung von HIV/Aids, Kinderarbeit, Polygamie, Opferrituale und Herrschaftsverhältnisse in einigen Ländern des Kontinents für sie eher noch mehr Fragen aufgeworfen als Möglichkeiten gezeigt, den afrikanischen Schülern auf Augenhöhe zu begegnen. Über die Lebensumstände der Jugendlichen wüssten die Theaterpädagogen noch viel zu wenig, weshalb sie es geradezu anmaßend fänden, während der Workshops im Wesentlichen Input zu geben. Um den Jugendlichen angemessen zu begegnen und sie wirklich da ,abzuholen', wo sie waren, sollte den Teenagern in den Workshops mehr Raum gewährt werden, sich zu äußern und ihre eigenen (Alltags-) Erfahrungen einzubringen.

\section{Anerkennung der lebensweltlichen Erfahrungen der Jugendlichen und Würdigung ihrer Mehrsprachigkeit}

Den zweiten Teil des Vorbereitungstreffens verbrachten die Theaterpädagogen damit Verfahren und Formen $\mathrm{zu}$ ersinnen und auszuprobieren, die die

\footnotetext{
${ }^{3}$ Indem die Schüler lernen, die Beiträge ihrer Klassenkameraden aufmerksam zu beobachten und sich ferner das Gesehene wechselseitig, in den jeweils besonderen Qualitäten beschreiben, bilden sie soziale Kompetenzen wie Einfühlungsvermögen aus und erweitern zugleich ihren Wortschatz.
} 
Jugendlichen selbst zu Wort kommen lassen und in denen sie ihre sprachliche und kulturelle Identität einbringen könnten. Die beiden wichtigsten Ergebnisse dieser Arbeitsphase waren erstens die morgendliche Frage des Tages und zweitens das Sprachporträt, das am Anfang einer jeden Werkstatt eingeführt und bearbeitet werden sollte: ${ }^{4}$ Die Frage des Tages, die jeden Morgen gestellt wird, kann sich auf ganz konkrete Informationen (Alter, Anzahl der Geschwister), aber auch auf Visionen, Lebensentwürfe, Träume, Ängste, Erlebnisse oder Meinungen zu bestimmten Themen beziehen, die dann im weiteren Verlauf des Workshoptages aufgegriffen, verhandelt und szenisch umgesetzt werden. Jeder und jede Teilnehmende - Theaterpädagogen und die Lehrkräfte inbegriffen - hat fünf bis zehn Minuten Zeit, um Gedanken zu der jeweiligen Frage zu entwickeln. In Gruppen stellen alle Teilnehmer nacheinander kurz ihre Antwort vor.

Was die Frageninhalte betrifft, so soll zunächst beim Allgemeinen angesetzt werden (Schulalltag, Deutschunterricht, strenge Eltern, Michael Jackson, Transportprobleme in Afrika) und erst dann werden mit viel Sensibilität über bestimmte Fragestellungen Unterschiede zwischen den Teilnehmenden ermittelt, wobei die Jugendlichen ihre Beiträge verbal oder spielerisch in verschiedensten Formensprachen formulieren dürfen. Bei der Moderation des sich anschließenden kurzen Gesprächs sollen die kulturellen und persönlichen Unterschiede als Reichtum anerkannt und gewürdigt werden.

Die Mehrheit der PASCH-Schüler spricht neben ihrer Muttersprache noch ein bis zwei regionale afrikanische Sprachen oder Dialekte sowie eine der Verkehrssprachen, also entweder Englisch oder Französisch. In seiner Unterscheidung zwischen einer teleologischen, einer gnoseologischen und eine kommunitären Dimension von Sprachlichkeit definiert Ehlich (2009) die gnoseologische Dimension als den Bereich, in dem Sprache Einfluss auf die Gedankenbildung eines Individuums nimmt. Er erklärt, dass bestimmte Erkenntnismöglichkeiten von Welt mit Hilfe einer jeweiligen Sprache gegeben sind, weshalb jede Sprache eine Weltansicht im Sinne Humboldts vorgibt. Es war also zu erwarten, dass die afrikanischen Teenager je nach Sprachhintergrund sehr unterschiedliche Denkweisen bzw. Weltansichten haben würden. Die kommunitäre Dimension von Sprache hat mit Identitätsbildung zu tun. Da sowohl die individuelle als auch die gesellschaftliche Identität über Sprache hergestellt wird, bestimmt und prägt nach Krumm (2008) insbesondere die Zugehörigkeit zur Sprachgemeinschaft der Erst- oder Familiensprache weitgehend auch das Wertesystem, die Religionszugehörigkeit, die Kommunikationskonventionen und die Körpersprache eines Menschen. Letzteres trifft für die Sprecher der über 1000 indigenen afrikanischen Sprachen sicherlich zu. Im afrikanischen Kontext lassen sich für Englisch- und Französischsprechende keine solchen Rückschlüsse auf Wertesystem oder Religionszugehörigkeit ziehen, da diese beiden Kolonialsprachen je nach lokalem Kontext auf sehr unterschiedliche Art und Weise mit den diversen Kulturen des Kontinents in Beziehung getreten

\footnotetext{
4 Eingehende Beschreibungen dieser Verfahren finden sich in dem SPRACH-FLUSSHandbuch (Holl 2011: 19, 53).
} 
sind. ${ }^{5}$

Die Theaterpädagogen beabsichtigten die jeweiligen Denkweisen im Sinne von Weltansicht und die der Identität jeweils zu Grunde liegenden Wertesysteme der Jugendlichen in den Workshops zu ermitteln und herauszustellen, um sich darüber in der szenischen Arbeit austauschen und kennenlernen zu können. Daher suchte das Team nach Übungen und Spielaufgaben, in denen die Teenager ihre jeweiligen Sprachen einbringen könnten, Aufgaben, die sie ermutigen würden, möglichst vielfältige Aspekte ihrer Persönlichkeit zu zeigen. Zudem reizte es die Workshopleiter, die Sprachenvielfalt als inspirierenden Fundus an Ausdrucksmitteln für den kreativen Prozess zu nutzen.

Hierzu bot sich das von Krumm (2001) entwickelte Sprachporträt an. Um ein Sprachporträt von sich zu erstellen, bekommen die Lernenden jeweils einen DIN A 4 Bogen mit einer Körpersilhouette. Zu jeder Sprache, die sie sprechen, assoziieren sie eine Farbe und markieren damit auf der Schemrenzeichnung, wo in ihrem Körper sie den Sitz der jeweiligen Sprache lokalisieren. Die fertigen Porträts werden einander vorgestellt und besprochen. Um aber die Skizzen, die der Bewusstmachung der persönlichen Sprachenkenntnisse dienen, dann wieder vom Papier zu lösen, sie körperlich spürbar, im Raum hörbar und damit szenisch nutzbar zu machen, haben die Theaterpädagogen ergänzende Übungen und Verfahren zum Sprachporträt entwickelt: Diese bestehen z.B. darin, dass die Jugendlichen

- Aufkleber mit der Bezeichnung der jeweiligen Sprachen anfertigen und sich diese dann an die entsprechenden Körperstellen kleben;

- wechselseitig aneinander die ,Sprachbuttons' auslösen, um sich - sozusagen per Knopfdruck - jeweils die verschiedenen Sprachen unterschiedlicher Partner anzuhören;

- bei ihrem Gegenüber Sprachknöpfe im Wechsel auslösen, um von dem Partner nacheinander Wortfolgen in verschiedenen Sprachen zu hören, die sich als rhythmisch ausgelöste Wortfolgen zu einer mehrsprachiger Wörterperkussion steigern lassen;

- die fremdsprachlichen Sätze ihrer Partner simultan mitsprechen;

- einander Passagen ins Ohr flüstern und während der „Einsager“ flüstert, der „Lautsprecher“ das Gesagte simultan nach außen hörbar macht.

Zum Abschluss ihres Vorbereitungstreffens stellten die Theaterpädagogen einen Kanon von zwölf gemeinsam ausprobierten, teils variierten, teils neu entwickelten Übungen und Verfahren zusammen, die bei den fünf Einführungsworkshops in Afrika angeleitet werden sollten. Dadurch sollte

\footnotetext{
${ }^{5}$ Auf den Begriff der Sprachgemeinschaft, der aus Sicht der Soziolinguistik problematisch ist, weil Sprachgemeinschaften keine homogenen Größen sind und ein Individuum stets Teil verschiedener Sprachgemeinschaften ist, kann an dieser Stelle nicht eingegangen werden.
} 
gewährleistet sein, dass alle Beteiligten des pan-afrikanischen Workshops in Johannesburg und des Begegnungsworkshops in Brandenburg über ähnliche Erfahrungen verfügen und dieselben Übungsformen und -prinzipien kennen. Jedem Workshopteam stand es frei, sich ein Thema oder einen Text für seine Werkstatt zu wählen. Festgelegt wurde lediglich, dass jeder Workshop mit einer kleinen Präsentation abschließen sollte. Die gemeinsam erarbeitete Aufführung als Ensemble einem Publikum zu präsentieren - diese inspirierende und motivierende Erfahrung sollten alle Jugendlichen machen dürfen.

\section{Die Workshops}

Windhoek, Lomé, Accra, Kampala, Nairobi und Johannesburg, das waren die Stationen der afrikanischen Workshops, an denen die Projektleiterin jeweils ihre drei anderen Kolleginnen und das dreiköpfige Filmteam aus Deutschland traf. Die Probenräume waren nicht immer ideal und wegen der Filmaufnahmen mussete in den tropischen Ländern zudem auf Ventilatoren oder Klimaanlage verzichtet werden. Die meisten der 20 Jugendlichen waren zum ersten Mal außerhalb ihres Landes und getrennt von ihren Familien, so dass bei der ersten Begegnung eine Mischung von Reisemüdigkeit, Unsicherheit, aber auch Spannung und Neugierde im Raum herrschte. Bei den ersten Kennlernspielen und der Begegnung mit der Kamera tauten die Jugendlichen schnell auf, übten fortan engagiert und erkundeten Spielaufgaben mit Elan und Interesse.

Irritationen und kulturelle Missverständnisse ereigneten sich meist in Situationen, die die Theaterpädagogen nicht antizipiert hatten. So auch bei der Übung Obst und Gemüse, bei der einer Person ein liebevolles Kompliment unter Nennung einer Frucht gemacht wird. Dazu geht A auf B zu, blickt diese Person freundlich an und haucht dann mit entzückter Stimme z.B. „B, du Pfirsich“. Dann lässt A sich - wie ein Vorhang - fallen und kauert sich vor B hin. B steigt nun mit einem großen Schritt über die kauernde Person A und geht nun ihrerseits zu einer Person, um dieser ein ,Obst-Kompliment' ${ }^{\prime} \mathrm{zu}$ machen. Die Theaterpädagogen hatten erwartet, dass hier der Blickkontakt zum Problem werden könnte, aber obwohl es in vielen Kulturen Afrikas als unhöflich oder sogar beleidigend gilt, einer Person in die Augen zu sehen, wollten die Teenager den direkten Blickkontakt gern im Spiel ausprobieren. Nicht so bei dem Hinkauern. Hier merkten die Theaterpädagogen schon beim Vormachen, wie viele Schüler hilfesuchend nach links und rechts schauten. Im spontanen Gespräch machte die Hälfte der Gruppe deutlich, dass das Übersteigen einer Person in ihren Kulturen - selbst im Spiel - niemals gemacht werden dürfte. Fortan wurde nach dem, Obst-Kompliment ' hinter der Person weggetaucht.

Die Fragen des Tages wurden zum täglichen Ritual. Die Einzelheiten über den Alltag der Jugendlichen, die Ideen, Meinungen und Vorlieben, mit denen die Teenager auf die Fragen reagierten, lieferten das Material, aus dem dann die Szenen entwickelt wurden. „Worum ging dein letzter großer Streit?" war z.B. eine Frage beim Workshop in Kampala, durch die herausgefunden werden sollte, was den Jugendlichen wirklich wichtig ist. Da die Teilnehmenden 
dieses Workshops lediglich über geringe Deutschkenntnisse verfügten, wurde ein Dialog vorgegeben, in den die Spielpartner zunächst ihre persönlichen Streiterfahrungen einsetzten, um die Repliken dann in verschiedenen Varianten zu proben. Dieser Szenenbeginn mit den wechselseitigen Anschuldigungen wurde mit einem Gang aufeinander zu kombiniert, während dessen sich die Partner in ihren jeweiligen Muttersprachen beschimpften. Der Auftritt endete in choreographierten Schlägereien, die aus ,Gemüse-Beschimpfungen' und aus (im Rahmen der Sprachporträts eingeführten) Wörterperkussion entwickelt worden waren. ${ }^{6}$

Bei ihren Workshops erlebten und erkannten die Theaterpädagogen, wie wichtig und bereichernd es für ihre Arbeit war, auch die Muttersprachen, die Zweit- und Drittsprachen sowie auch alle von den Jugendlichen gesprochenen Varietäten einzubeziehen, um die Kommunikationskompetenzen und die Ausdrucksfähigkeit der Teenager auszubilden. Dazu ein Beispiel: Bei dem Workshop in Windhoek nahm Nonzuzo, ein 14-jähriges Mädchen aus KwaZuluNatal, teil. Sie gab sich sehr zurückhaltend, sprach besonders leise, kulturbedingt nahm sie nie Blickkontakt auf, initiierte keine Gespräche und beteiligte sich nur, wenn sie direkt angesprochen wurde. Auch bei den ersten Gruppenübungen und Spielen machte sie eher einen gehemmten Eindruck, als wäre ihr Vieles unangenehm. Am zweiten Tag dann beschäftigten wir uns mit dend Sprachporträts, wobei sich herausstellte, dass Nonzuzo vier Sprachen sprechen und sich sehr sensibel in andere Sprachen einfühlen und diese simultan mit großer phonetischer Präzision mitsprechen konnte. Als sie dann die Aufgabe bekam, uns den Gang ihrer Großmutter vorzumachen und zu zeigen, wie diese Gogo mit ihr schimpft, walzte sie plötzlich als wütende Oma durch den Raum und setzte unter großem Gestikulieren zu einer unglaublich lauten Schimpftirade an. Die Figur der ungestümen Zulu-Oma wurde gleich von allen Teilnehmenden begeistert aufgenommen und nachgespielt. Nonzuzo wiederholte den extremen Oma-Auftritt mit Gang, Gesten und Lautstärke - diesmal aber auf Deutsch. Der Umweg über die Muttersprache ermutigte Nonzuzo, völlig neue Aspekte ihrer Persönlichkeit zu zeigen. Fortan beteiligte sie sich rege, initiierte Gesprächskontakte, reagierte auf die Anderen und nutzte Körpersprache und ihr umfangreiches stimmliches Ausdruckrepertoire zum Kommunizieren.

Besonders in Afrika, wo in Folge der oft großen Klassenstärken und des sehr reglementierten Unterrichtsstils wenig Platz ist, Individuelles zu entfalten und zu entwickeln, lohnte es sich, die Jugendlichen in der Theaterarbeit und im Unterricht ganzheitlich anzusprechen und ihnen die Gelegenheit zu bieten, möglichst viele Facetten ihrer Persönlichkeit einzubringen und zu entfalten.

\footnotetext{
${ }^{6}$ Um zu verhindern, dass sich beim Üben falsche sprachliche Wendungen einschliffen, überprüften die Theaterpädagogen vor jeder Probenphase alle Beiträge und Dialoge der Lernenden auf Sprachrichtigkeit.
} 


\section{Zwischenfazit Workshops}

Aus Zeitgründen und auf Grund der weiten Entfernungen konnte keine gemeinsame abschließende Auswertung der Workshops in der großen Runde der Theaterpädagogen und Lehrkräfte stattfinden. Nach jedem Workshop aber setzen sich die Pädagogen zusammen um festzustellen, inwiefern die Jugendlichen von der theaterpädagogischen Arbeit profitiert hatten. Eine quantitative Überprüfbarkeit der hinzugewonnenen sozialen, sprachlichen und kommunikativen Kompetenzen war nicht möglich, denn eine Abschlussprüfung gab es nicht, zumindest aber beschrieben die Beteiligten der Auswertungsrunde qualitativ, welche Fähigkeiten und Fertigkeiten die Jugendlichen insgesamt bei den Workshops erworben hatten. Hinsichtlich der kommunikativen und der interkulturellen Kompetenzen wurde hinzu gewonnenes Selbstvertrauen bemerkt und benannt, mit dem sich auch schüchterne Persönlichkeiten neuerdings in der Gruppe und vor dem Publikum präsentierten. Beobachtet wurde auch die Fähigkeit, entspannt in mehrsprachigen Gruppen zu agieren, Kommunikationssituationen besser einschätzen und Gesprächspartner und Subtext besser lesen zu können und durch die erworbene Kongruenz von Körpersprache und Aussage klarer mit Gesprächspartnern unterschiedlich-kulturellen Hintergrunds interagieren zu können. Die mündliche Ausdrucksfähigkeit sämtlicher Teenager war merklich differenzierter und gewandter geworden. Die Aussprache und Intonation der Lernenden hatte sich verbessert, die Schüler sprachen flüssiger, ihr Sprechtempo hatte sich gesteigert und die Verknüpfungen von Sätzen gelangen eleganter. Alle Lehrkräfte bestätigten zudem, dass der Wortschatz der Lernenden sich erheblich vergrößert hatte und dass ihren Schülern was Satzbau und Wortwahl anbelangte, merklich weniger Fehler unterliefen als noch zu Beginn des siebentägigen Workshops.

Inwiefern sich im Verlauf eines Workshops jeweils die Persönlichkeit der Teilnehmenden erweitert und ausgebildet hat, lässt sich hier nicht im Einzelnen beurteilen. Aus den Beobachtungen von acht Workshops mit über 130 Teenagern zeigt sich jedoch folgender Zusammenhang zwischen wahrnehmbarer Weiterentwicklung der Schülerpersönlichkeiten und dem auf Mehrsprachigkeit setzenden theaterpädagogischen Prozess:

Beim Ergründen der eigenen Mehrsprachigkeit mittels der Sprachporträts und den weiterführenden Verfahren dazu erlebten viele der SPRACH-FLUSSTeilnehmenden - und zwar auch die in der Bundesrepublik Ansässigen zum ersten Mal in ihrem Leben, dass sich jemand für ihre Mehrsprachigkeit interessierte und ihre zum Teil außerordentlichen Sprachenkenntnisse würdigte. Durch diese Sprachbewusstheit (und es ist nicht übertrieben, dass vielen Schüler beim Erstellen der Sprachporträts eine Art Bewusstseinserlebnis zuteil wurde) erlebten die Jugendlichen, dass der theaterpädagogische Prozess unmittelbar mit ihnen selbst zu tun hatte. Infolge der Würdigung ihres sprachlichen Hintergrunds und des aufmerksamen Interesses an ihren lebensweltlichen Erfahrungen, Kenntnissen und Idealen wurde den Jugendlichen eine Anerkennungserfahrung zuteil. Solche Formen der Anerkennung sind, 
wie Stojanov (2006) zeigt, die Voraussetzung für die Selbst-Entwicklung und Welt-Erschließung eines Individuums, sie fördern individuelle Autonomie und befähigen erst zur sozialen Partizipation.

\section{Machtdiskurs vs. interkultureller Dialog}

In den auswertenden Gesprächen berichteten die Leiter der verschiedenen Workshops über Erlebnisse und Begebenheiten während der SPRACH-FLUSS-Arbeit, die ihnen bewusst gemacht hätten, dass die Entscheidung, Mehrsprachigkeit nicht auszublenden und zu verbieten, sondern Sprachenvielfalt im Sinne eines interkulturellen Dialogs in der theaterpädagogischen Arbeit zu fördern, immer auch eine politische Dimension hat.

Ausgehend von einer Schüleräußerung beim Workshop in Nairobi wird nachstehend ausgeführt, wie die Folgen von Machtausübung durch Sprache noch heute im postkolonialen Afrika spürbar sind. Es wird dezidiert auf dieses afrikanische Beispiel eingegangen, weil sich daraus Parallelen zu diskriminierender Sprachpolitik in Deutschland und anderen Einwanderungsländern Europas ziehen lassen: In Nairobi lautete eine Frage des Tages: „Wie möchtest du mit 27 sein?" Die Jugendlichen stellten einander ihre Zukunftsvisionen vor. Als Louisa aus Kamerun, Berufswunsch Ärztin, an der Reihe war, kam es zu folgendem Dialog zwischen ihr und Njerie aus Kenia, die Juristin werden möchte:

Njerie: Sind Männer und Frauen bei euch in Kamerun gleichberechtigt?Louisa: (nickt bestätigend) Ja, ja.Njeerie: Aber es gibt doch Polygamie bei euch.Louisa: Ja, viel.Njerie: Haben die Frauen bei euch auch mehrere Männer?Louisa: (lacht) Nein, natürlich nicht.Louisa ist eine sprachbegabte Schülerin, die problemlos das Adjektiv gleichberechtigt mit dem Verb sein konstruieren kann. Wie kommt es, dass sie die polygame Praxis in Kamerun mit der Gleichberechtigung der Geschlechter vereinbaren kann?

EineinteressanteErklärung hierfürliefert die senegalesische Germanistikprofessorin Khadi Fall. In ihrem Aufsatz „Sprache, Machtdiskurs und Machtlosigkeit“ (2008) beschreibt sie, wie 1960 mit der Unabhängigkeit Senegals von der neuen Regierung um Léopold Sédar Senghor Französisch als offizielle Landessprache eingeführt wurde und zwar auf Kosten der westafrikanischen Kommunikationssprachen - allen voran des von 90 Prozent der Bevölkerung gesprochenen Wolof.

Französisch wurde in den Kolonialschulen mit der den Franzosen eigenen Vehemenz eingeführt, Kinder, die sich in diesen Schulen weiterhin auf Wolof unterhielten, wurden bloßgestellt, angeprangert, bestraft, suspendiert. (Am SPRACH-FLUSS-Projekt beteiligte Jugendliche bestätigten, dass es noch heute in vielen Ländern der Frankophonie verboten ist, die indigenen Muttersprachen zu sprechen.) Dennoch waren die senegalesischen Eliteschulen begehrt, da sie Studienaufenthalte in Frankreich ermöglichten und die Aussichten auf einen wohl dotierten Posten in der Regierung der jungen Republik erhöhten. 
An allen Schulen Senegals wurde Französisch nach der am altsprachlichen Unterricht orientierten méthode classique unterrichtet, einem von außen gesteuerten, frontal organisierten Unterrichtsprinzip, welches der GrammatikÜbersetzungs-Methode ähnlich ist. Im Zentrum des Unterrichts standen Texte aus Frankreich; einfach ausgeblendet wurden der lokale kulturelle Kontext der afrikanischen Lernenden mit den Gegebenheiten ihres Lernumfelds, die Wertegemeinschaft, der sie angehörten, und auch die lokalen Sprachen in der Klasse .

Diese Sprachpolitik hat weit reichende Folgen für die gegenwärtige senegalesische Gesellschaft. Laut Fall ist die Bevölkerung des Landes noch heute gespalten in einerseits die im französischen Bildungssystem ausgebildete Elite, die lediglich 30 Prozent der Bevölkerung ausmacht, aus der sich jedoch die gesamte Regierung rekrutiert, und andererseits die restlichen 70 Prozent der Bevölkerung, die lediglich die normalen Schulen besuchen konnten. Diese Mehrheit ist nicht an den auf Französisch geführten Diskursen der Elite über republikanische Institutionen und Rechte und Pflichten von Staatsbürgern interessiert.

Der Großteil der senegalesischen Bevölkerung will von den politischen Debatten im eigenen Staat nichts wissen, weil diese nichts mit der Lebenswirklichkeit im Land zu tun haben. Selbstverständlich spricht man überall im Senegal Französisch, aber Begriffe, Konzepte und Werte, die die Gesellschaft und das Staatswesen betreffen, wie z.B. Demokratie, Bürger, republikanische Gesetze bleiben für die meisten Senegalesen Worthülsen - weil sie nicht mit den Traditionen, Bräuchen und Werten der afrikanischen Kulturen zu vereinbaren sind.

Bei einer 2009 durchgeführten Untersuchung im Rahmen des Afrobarometer, zu der 25.000 Bürger aus 18 afrikanischen Ländern von Benin bis Simbabwe repräsentativ (in ihren jeweiligen Muttersprachen) befragt wurden, was sie sich jeweils unter Demokratie vorstellten, kam heraus, dass nur etwa 6 Prozent der Befragten mit Demokratie in erster Linie ein Mehrparteiensystem verbinden (cf. Wiedermann 2009).

Auch im Fall Louisas verhält es sich so, dass sie wohl weiß, was das deutsche Wort Gleichberechtigung bedeutet, das Wertesystem, in welches sie hineinsozialisiert worden ist, ihre Weltansicht, erschwert es ihr jedoch, den Begriff auf die gesellschaftlichen Verhältnisse in ihrem Land anzuwenden.

Die Sprachpolitik im Senegal, die eine Amtssprache von oben verordnet hat, die zudem unterrichtet wurde, ohne dabei die kulturellen Orientierungssysteme der Lernenden zu berücksichtigen, hat dazu geführt, dass die meisten Senegalesen das politische und ökonomische System, in dem sie leben, nicht als das ihre begreifen. Besonders folgenreich ist es, wenn jungen Menschen sich nicht mit Regierung und Staat identifizieren können. Ihnen fehlt damit nämlich auch die Bedingung für die Möglichkeit, Perspektiven und Visionen für ihre Zukunft im eigenen Land zu entwerfen und zu entwickeln. Fall (2008) stellt hier einen direkten Zusammenhang zwischen entfremdender Sprachpolitik und Flucht aus dem eigenen Land her und verweist auf die vielen jungen Senegalesen, die 
auf den seeuntüchtigen Piirogen der Fischer ihr Leben riskieren, um über das Mittelmeer nach Europa zu gelangen, wo sie sich eine bessere Zukunft erhoffen.

Sprachpolitik, dienichtaufinterkulturellen Austausch setzt, grenzt Individuen bestimmter Sprachgruppen aus, was wiederum zur Spaltung von Gruppen und ganzen Gesellschaften führen kann. Solche Teilung ereignet sich nicht nur im Senegal, sondern in vielen Ländern der Welt und sehr oft auch in deutschen Klassenzimmern: An vielen Schulen Deutschlands wird die Mehrsprachigkeit von Schülern mit Migrationshintergrund in erster Linie als Defizit im Hinblick auf die Zielsprache Deutsch gesehen. Gogolin (1994) spricht - auf den sprachlichen Aspekt hinweisend - vom „monolingualen Habitus der multikulturellen Schule“, und Interviews mit „ausgesiedelten“ Schülern (cf. Schanz/Röder 1995) zeigen, wie mit dem Ausblenden ihres sprachlichen Hintergrunds von diesen Lernenden verlangt wird, dass sie im Schulalltag solche Anteile ihrer Persönlichkeit abspalten, die mit ihren Herkunftskulturen und den damit verbundenen Weltansichten zu tun haben. Diese Schüler werden dadurch zu eindimensionalen Persönlichkeiten degradiert und in der Konsequenz auch als solche behandelt, was wiederum dazu führt, dass viele Jugendliche die ihnen zugewiesene ,eindimensionale Rolle annehmen - nicht nur als Individuen, sondern auch als Gruppe.

Mehrere SPRACH-FLUSS-Theaterpädagogen, die sogenannte IntegrationsProjekte leiten, bestätigten wie kompliziert und problematisch es sei, Teenager $\mathrm{zu}$ erreichen und einzubeziehen, für deren sprachlichen und kulturellen Hintergrund sich noch nie jemand interessiert hat.

Die vielleicht wichtigste Erkenntnis, die die Theaterpädagogen aus den SPRACH-FLUSS-Workshops gewonnen haben, ist, dass Mehrsprachigkeit eine kulturelle und interkulturelle Herausforderung für ihre Arbeit darstellt, nämlich theaterpädagogische Mittel und Wege zu finden, um über ein wechselseitiges Verstehen der verschiedenen Sprachen hinaus auch ein Verständnis der unterschiedlichen Denkweisen der Beteiligten zu erlangen. Die SPRACHFLUSS Workshops haben gezeigt, mit welchen theaterpädagogischen Mitteln ein kollektiver kreativer Prozess initiiert und gefördert werden kann, bei dem die einzelnen Jugendlichen ihre Mehrsprachigkeit einbringen können und dadurch ermutigt werden, ihre eigenen lebensweltlichen Erfahrungen, Meinungen und Ideen verbal oder spielerisch in verschiedenen Formensprachen zu formulieren, so dass ihre (kostbaren) kulturbedingten Unterschiede wechselseitig anerkannt, gewürdigt, verhandelt und verstanden werden können.

\section{SPRACH-FLUSS im Deutschunterricht}

In dem SPRACH-FLUSS-Handbuch sind 30 theaterpädagogische Übungen und Verfahren zusammen gestellt, die gemeinsam mit Schülern und Lehrkräften in den Probenräumen der afrikanischen Workshops ausprobiert worden sind. Etliche Übungen wurden zudem abgewandelt und variiert, damit sie auch im Deutschunterricht mit großen Lerngruppen eingesetzt werden können. 
Wie wirksam es ist, die bei SPRACH-FLUSS entwickelten theaterpädagogischen Übungen und Verfahren für das Deutschlernen einzusetzen, zeigt sich z.B. an dem erfolgreichen Intensivkursformat, welches die Verfasserin am GoetheInstitut Johannesburg für Gruppen von Stipendiaten und Geschäftsleuten aus ganz Subsahara Afrika anbietet. ${ }^{7}$ Bei diesem vierwöchigen Kurs (160 UE) wird die Arbeit am Lehrwerk im Klassenraum kombiniert mit theaterpädagogischen Übungen, Spielen, chorischen Aufgaben, Sprachporträts, szenischen Dialogen und Fragen des Tages, die in einem gesonderten Probenraum eingeführt bzw. geprobt werden. Zwischen 75 und 90 Prozent der Lernenden, die zuvor keine Deutschkenntnisse haben, bestehen nach nur einem Kursmonat die Start-Deutsch-1 Prüfung (A1).

In Workshops und Seminaren des Goethe-Instituts wurden afrikanische Deutschlehrkräfte dazu angeregt, ebenfalls theaterpädagogische Mittel in ihrem Unterricht zu nutzen. Schon auf den entsprechenden Lehrerfortbildungen zeigte sich, dass die meisten Übungen und Verfahren, die aus dem kollektiven ästhetischen Prozess im Probenraum adaptiert worden waren, in der räumlich (und zeitlich) begrenzten Unterrichtssituation im Klassenzimmer ihr Ziel und ihren Charakter veränderten:

Die Frage des Tages, die im offenen kreativen Prozess dazu dient, den Erfahrungsraum der Lernenden auszuleuchten, wird im Deutschunterricht zur Vorentlastung von neuen Texten eingesetzt oder um in das jeweilige Thema einer Lektionen einzuführen.

Obst und Gemüse, die Übung, mit der im Probenraum die Ausdrucksfähigkeit der Jugendlichen und die Kongruenz von Sprachhaltung und verbaler Aussage geprobt wird, verschiebt sich im Klassenkontext hin zu einer Wortschatz- und Grammatikübung, bei der die Deklination der Adjektive („Naba, du sanfter Pfirsich!") eingeübt wird.

Sprachporträts, die die Sprachenvielfalt im Probenraum hörbar werden lassen und damit einen inspirierenden Fundus an Ausdrucksmöglichkeiten als Ausgangspunkt für die szenische Arbeit liefern, dienen in einer Deutschstunde lediglich zur Abfrage und Ermittlung der in der Lerngruppe gesprochenen Sprachen.

Dialoggerüste, aus denen die Jugendlichen eigenständig Szenen entwickeln, indem sie ihre persönlichen Erfahrungen und Belange einsetzen, werden im Deutschunterricht zu Loops (cf. Dockalova 2011) oder Chunks, also „als Ganzes gespeicherte zielsprachliche Sequenzen“ (Aguado 2009), die von Lehrkräften eingeführt werden, um jeweils bestimmte lexikalisch-grammatischen Phänomene einzuüben.

Der gezielte Einsatz dieser theaterpädagogischen Mittel im (lehrwerksgestützten) Deutschunterricht hat sich in vielfacher Hinsicht bewährt. Ansätze und Verfahren, die sich bei den SPRACH-FLUSS-Workshops als besonders effektiv erwiesen haben, werden von Deutschlehrkräften, die in anderen Zusammenhängen arbeiten, bestätigt. So liefert z.B. Cieslak (2010) in ihrem

\footnotetext{
${ }^{7}$ Vgl. die Website des Goethe-Instituts Südafrika: http://www. goethe.de/ins/za/joh/l rn/ deu/de8332008.htm
} 
Plädoyer für die Vorgehensweise „Vom Text zum Bild“ die Begründung dafür, dass es sinnvoller ist, in die Lektion eines Lehrwerks mit einer Frage des Tages einzuführen, zu der die Lernenden eigene Bilder assoziieren und verbalisieren müssen, als - wie in den Werken oft vorgesehen - mit Bildern und Bildcollagen zu beginnen, da diese die Imagination der Lernenden einschränken.

Dass theaterpädagogische Mittel, wenn sie gezielt im Grammatikunterricht eingesetzt werden, Inhalte lebendig und sinnhaft werden lassen und eine wirksame Verarbeitung grammatischer Strukturen und Phänomene gewährleisten, bestätigen Erfahrungsberichte von Lehrkräften bei Fortbildungen und auf Deutschlehrerkonferenzen. Die Vielzahl von Veröffentlichungen und Lehrerhandbüchern im Schnittfeld von Theaterpädagogik und Fremdsprachenunterricht ${ }^{8}$, die in den letzten zehn Jahren stark zugenommen hat, legt nahe, dass theaterpädagogische Mittel sich im Deutschunterricht wachsender Beliebtheit erfreuen.

Der grundlegende Unterschied zwischen einem offenen kreativen Prozess im Probenraum, der in eine gemeinsame Präsentation mündet, und den punktuell und gezielt eingesetzten Übungen im Klassenzimmer, mit denen ein bestimmtes grammatikalisches Phänomen verarbeitet und vertieft werden soll, zeigt sich darin, ob und wie das (Spiel)Material generiert wird: Während die Dialoggerüste in der kollektiven kreativen Praxis Lernende dazu anregen, eigene, auch mehrsprachige Szenen zu entwickeln und zu spielen, also Szenen, die mit ihrer Lebenswelt zu tun haben, geht es z.B. den Autorinnen von Chunks für DaF um die „Versorgung der Lehrenden und Lernenden mit [deutschen, Anm.d.Verf.] Chunk-Angeboten einerseits, mit Analyserastern und aufbereiteten Regeln andererseits" (Handwerker/Madlener 2009:17). Während einzelne theaterpädagogische Mittel oder Verfahren, mit denen Lehrkräfte im Hinblick auf das Einüben bestimmter sprachlicher Strukturen ausgestattet werden, ohne weiteres im Rahmen einer Unterrichtsstunde eingeführt und ausprobiert werden können, lassen sich die an der Mehrsprachigkeit der Jugendlichen ansetzenden, kreativen theaterpädagogischen Prozessen mit Schülergruppen unterschiedlicher Kulturen, die in einer Präsentation münden sollen, nicht so einfach in den Schulkontext integrieren. Da gibt es zeitliche Einschränkungen: Ein sechstätiger Workshop kann nur außerhalb des normalen Stundenplans stattfinden. Raumprobleme treten auf, denn in den meisten Schulen ist kein Probenraum über einen so langen Zeitraum verfügbar. Es kommt zu Koordinationsschwierigkeiten, wenn eine klassenübergreifende oder gar schulübergreifende Lerngruppe für einen Workshop freigestellt werden muss. Schließlich gibt es unter den Lehrkräften an Schulen nur wenige im interkulturellen Dialog erfahrene Theaterpädagogen/innen, die ein solches Projekt gemeinsam konzipieren, organisieren und zu einer Präsentation führen können. Selbst wenn es einzelne Lehrkräfte gäbe, ist es meistens ein Problem, diese über einen längeren Zeitraum freizustellen. Aus diesen Gründen werden an Schulen nur selten prozesshafte Workshops angeboten, bei denen die Teilnehmenden ermutigt werden, ihre eigene sprachliche und kulturelle Identität

\footnotetext{
${ }^{8}$ Vgl. z.B. Even (2003, 2011), Tselikas (1999), Imkamp (1996), Schewe (1998).
} 
$\mathrm{zu}$ erforschen, sie wechselseitig anzuerkennen und in einen interkulturellen Dialog mit anderskulturellen Altersgenossen zu treten.

Aus der Überzeugung heraus, dass Theatermittel im Deutschunterricht unverzichtbar sind, dass aber darüber hinaus viel mehr Kinder und Jugendliche von prozesshaften, mehrsprachigen Werkstätten profitieren sollten, entstand das Konzept PROBE! - mobile Teams für kreative Lernwege. Auf ihrer Website ${ }^{9}$ präsentieren sich interkulturell erfahrene Theaterpädagogen und Lehrkräfte (z.T. aus dem Projekt SPRACH-FLUSS), die als PROBE!-Teams die Zusammenarbeit mit Schulen, Universitäten und kulturellen Bildungsstätten suchen. Nach Maßgabe der jeweiligen Institution konzipieren, organisieren und leiten diese PROBE!-Teams Werkstätten im Sinne von SPRACH-FLUSS, um Kinder und Jugendliche unterschiedlicher kultureller Herkunft ,da abzuholen, wo sie sind'.

\section{Bibliografie}

Aguado, Karin (2009): Chunks - nur Bausteine kompetenter Sprachverwendung oder auch Katalysatoren lernersprachlicher Entwicklung? Handreichung zum Vortrag auf der XIV. Internationales Deutschlehrertagung in Jena

http://karin.aguado.de/publ/downloads/aguado-chunks-idt-jena2009_08_06.pdf (19.01.2012)

Cieslak, Renata (2010): Vom Text zum Bild - Dramapädagogische Methoden im DaF-Unterricht. In: Scenario 2010/1

http://publish.ucc.ie/scenario/2010/01/cieslak/06/de

Dockalova, Bara (2011): Loops - A multi-purpose drama technique fort the language classroom. In: Scenario 2011/1

http://publish.ucc.ie/scenario/2011/01/dockalova/06/en

Ehlich, Konrad (2009): Sprachenpolitik in Europa - Tatsachen und Perspektiven. In: Stellenbosch Papers in Linguistics PLUS 38, Multilingualism and language policies in Africa, 6-41

Even, Susanne (2011): Studiosus congens und studiosus ludens - Grammatik inszenieren. In: Küppers, Almut;Schmidt, Torben; Walter, Maik: Inszenierungen im Fremdsprachenunterricht. Braunschweig: Diesterweg 68-92

Even, Susanne (2003): Drama Grammatik. Dramapädagogische Ansätze für den Grammatikunterricht Deutsch als Fremdsprache. München: iudicium

Fall, Khadi (2008): Sprache, Machtdiskurs und Machtlosigkeit. In: Limbach, Jutta; von Ruckteschell, Katharina (Hrsg.): Macht der Sprache. München: Langenscheidt, 63-67

Gogolin, Ingrid 1994: Der monolinguale Habitus der multilingualen Schule. Münster/New York 1994: Waxmann Verlag

9 www.kreative-lernwege.com 
Handwerker, Brigitte; Madlener, Karin (2009): Chunks für DaF. Theoretischer Hintergrund und Prototyp einer multikulturellen Lernumgebung. Hohengehren: Schneider

Holl, Edda (2011): SPRACH-FLUSS, Theaterübungen für Sprachunterricht und interkulturelles Lernen. Ismaning: Hueber Verlag

Hufeisen, Britta (2008): Wieso ist „Mehrsprachigkeit“ ein solch aktuelles Schlagwort? In: Frühes Deutsch 14/2008, 4-7

Imkamp, Judith-Mira (1996): Spielerische Unterrichtshilfen. Pilsen: Westböhmische Universität

Krumm, Hans-Jürgen (2008): Sprache und Identität. In: Limbach, Jutta; von Ruckteschell, Katharina (Hrsg.): Macht der Sprache. München: Langenscheidt, 29-30

Krumm, Hans-Jürgen (2001): Kinder und ihre Sprachen - lebendige Mehrsprachigkeit. Wien: Eviva

Rubin, Joan (1975): What the 'Good Language Learner' Can Teach Us. In: TESOL Quarterly 9, 41-51

Sarter, Heidemarie (1997): Fremdsprachenarbeit in der Grundschule. Neue Wege - neue Ziele. Darmstadt: Wissenschaftliche Buchgesellschaft

Schanz, Claudia; Röder, Uta (1995): Anwesend, aber nicht zugehörig sein. Gespräch mit zwei ausgesiedelten Jugendlichen. In: Pädagogik 10, 14-17

Schewe, Manfred (1998): Dramapädagogisch lehren und lernen. In: Jung, Udo O.H. (Hrsg.): Praktische Handreichung für Fremdsprachenlehrer. Frankfurt am Main: Peter Lang, 334-340

Stojanov, Crassimir (2006): Bildung und Anerkennung. Soziale Voraussetzungen von Selbst-Entwicklung und Welt-Erschließung. Wiesbaden: VS Verlag für Sozialwissenschaften

Tselikas, Elektra I. (1999): Dramapädagogik im Sprachunterricht. Zürich: Orell Füssli Verlag

Wiedermann, Charlotte (2009): Gelebte Demokratie. Was es heißt Bürger zu sein in Afrika. In: Le Monde diplomatique 5, 10-15 